\title{
Metastatic Thymic Carcinoma
}

National Cancer Institute

\section{Source}

National Cancer Institute. Metastatic Thymic Carcinoma. NCI Thesaurus. Code C148128.

Thymic carcinoma which has spread from the original site of growth to another anatomic site. 